\title{
Complete genome sequence of the moderately thermophilic mineral-sulfide-oxidizing firmicute Sulfobacillus acidophilus type strain (NAL ${ }^{\top}$ )
}

\author{
Iain Anderson ${ }^{1}$, Olga Chertkov ${ }^{1,2}$, Amy Chen ${ }^{3}$, Elizabeth Saunders ${ }^{1,2}$, Alla Lapidus ${ }^{1}$, Matt \\ Nolan', Susan Lucas', Nancy Hammon ${ }^{1}$, Shweta Deshpande', Jan-Fang Cheng ${ }^{1}$, Cliff Han ${ }^{1,2}$, \\ Roxanne Tapia ${ }^{1,2}$, Lynne A. Goodwin ${ }^{1,2}$, Sam Pitluck ${ }^{1}$, Konstantinos Liolios ${ }^{1}$, Ioanna Pagani ${ }^{1}$, \\ Natalia Ivanova', Natalia Mikhailova ${ }^{1}$, Amrita Pati ${ }^{1}$, Krishna Palaniappan ${ }^{3}$, Miriam Land ${ }^{1,4}$, \\ Chongle Pan ${ }^{1,4}$, Manfred Rohde ${ }^{5}$, Rüdiger Pukall ${ }^{6}$, Markus Göker ${ }^{6}$, John C. Detter ${ }^{1,2}$, Tanja \\ Woyke $^{1}$, James Bristow ${ }^{1}$, Jonathan A. Eisen ${ }^{1,7}$, Victor Markowitz ${ }^{3}$, Philip Hugenholtz ${ }^{1,8}$, \\ Nikos C. Kyrpides ${ }^{1}$, Hans-Peter Klenk ${ }^{6 *}$, and Konstantinos Mavromatis ${ }^{1}$ \\ ${ }^{1}$ DOE Joint Genome Institute, Walnut Creek, California, USA \\ ${ }^{2}$ Los Alamos National Laboratory, Bioscience Division, Los Alamos, New Mexico, USA \\ ${ }^{3}$ Biological Data Management and Technology Center, Lawrence Berkeley National \\ Laboratory, Berkeley, California, USA \\ ${ }^{4}$ Oak Ridge National Laboratory, Oak Ridge, Tennessee, USA \\ ${ }^{5} \mathrm{HZI}$ - Helmholtz Centre for Infection Research, Braunschweig, Germany \\ ${ }^{6}$ Leibniz Institute DSMZ - German Collection of Microorganisms and Cell Cultures, \\ Braunschweig, Germany \\ ${ }^{7}$ University of California Davis Genome Center, Davis, California, USA \\ ${ }^{8}$ Australian Centre for Ecogenomics, School of Chemistry and Molecular Biosciences, The \\ University of Queensland, Brisbane, Australia
}

*Corresponding author: Hans-Peter Klenk (hpk@dsmz.de)

Keywords: aerobic, motile, Gram-positive, acidophilic, moderately thermophilic, sulfide- and iron-oxidizing, biomining, autotrophic, mixotrophic, soil, insertis sedis, Clostridiales, GEBA

Sulfobacillus acidophilus Norris et al. 1996 is a member of the genus Sulfobacillus which comprises five species of the order Clostridiales. Sulfobacillus species are of interest for comparison to other sulfur and iron oxidizers and also have biomining applications. This is the first completed genome sequence of a type strain of the genus Sulfobacillus, and the second published genome of a member of the species $S$. acidophilus. The genome, which consists of one chromosome and one plasmid with a total size of 3,557,831 bp harbors 3,626 proteincoding and 69 RNA genes, and is a part of the Genomic Encyclopedia of Bacteria and Archaea project.

\section{Introduction}

The genus Sulfobacillus currently consists of five species [1], all of which are mildly thermophilic or thermotolerant acidophiles [2]. Sulfobacilli grow mixotrophically by oxidizing ferrous iron, sulfur, and mineral sulfides in the presence of yeast extract or other organic compounds [3]. Some can also grow autotrophically $[2,3]$. The strains that have been tested are capable of anaerobic growth using $\mathrm{Fe}^{+3}$ as an electron acceptor [2,4]. The genus Sulfobacillus, along with the genus Thermaerobacter, have only tentatively been assigned to a family, "Clostridiales
Family XVII incertae sedis". This group may form a deep branch within the phylum Firmicutes or may constitute a new phylum [5]. Strain $\mathrm{NAL}^{\mathrm{T}}$ (= DSM $10332=$ ATCC 700253) is the type strain of the species Sulfobacillus acidophilus. The genus name was derived from the Latin words 'sulfur' and 'bacillus' meaning 'small sulfur-oxidizing rod' [6]. The species epithet is derived from the Neo-Latin words 'acidum', acid, and 'philus', loving, meaning acidloving [3]. The first genome from a member of the species $S$. acidophilus, strain TPY, which was isolated 
from a hydrothermal vent in the Pacific Ocean, was recently sequenced by Li et al. [7]. Here we present a summary classification and a set of features for $S$. acidophilum strain NALT, together with the description of the complete genomic sequencing and annotation.

\section{Classification and features}

A representative genomic 16S rRNA sequence of $S$. acidophilus $\mathrm{NAL}^{\mathrm{T}}$ was compared using NCBI BLAST $[8,9]$ under default settings (e.g., considering only the high-scoring segment pairs (HSPs) from the best 250 hits) with the most recent release of the Greengenes database [10] and the relative frequencies of taxa and keywords (reduced to their stem [11]) were determined, weighted by BLAST scores. The most frequently occurring genera were Sulfobacillus (81.9\%), Thermaerobacter (8.0\%), Laceyella (2.8\%), 'Gloeobacter' (2.1\%) and 'Synechococcus' (2.0\%) (76 hits in total). Regarding the six hits to sequences from members of the species, the average identity within HSPs was 98.9\%, whereas the average coverage by HSPs was $97.2 \%$. Regarding the 23 hits to sequences from other members of the genus, the average identity within HSPs was $93.1 \%$, whereas the average coverage by HSPs was $81.2 \%$. Among all other species, the one yielding the highest score was "Sulfobacillus yellowstonensis" (AY007665), which corresponded to an identity of $99.4 \%$ and an HSP coverage of $97.0 \%$. (Note that the Greengenes database uses the INSDC (= EMBL/NCBI/DDBJ) annotation, which is not an authoritative source for nomenclature or classification.) The highest-scoring environmental sequence was HQ730681 ('Microbial Anaerobic Sediments Tinto River: Natural Acid and Heavy Metals Content extreme acid clone SN1 2009 12D'), which showed an identity of $94.5 \%$ and an HSP coverage of $99.0 \%$. The most frequently occurring keywords within the labels of all environmental samples which yielded hits were 'acid' (4.8\%), 'soil' (4.5\%), 'hydrotherm' (3.7\%), 'microbi' (3.7\%) and 'mine' (3.0\%) (172 hits in total). These keywords correspond well to the environment from which strain NAL ${ }^{\mathrm{T}}$ was isolated. Environmental samples that yielded hits of a higher score than the highest scoring species were not found.

Figure 1 shows the phylogenetic neighborhood of S. acidophilus NAL ${ }^{\mathrm{T}}$ in a $16 \mathrm{~S}$ rRNA based tree. The sequences of the five $16 \mathrm{~S}$ rRNA gene copies in the genome differ from each other by up to eight nucleotides, and differ by up to four nucleotides from the previously published 16S rRNA sequence (AB089842), which contains two ambiguous base calls.

Cells of $S$. acidophilus NAL $^{\mathrm{T}}$ are rods 3.0-5.0 $\mu \mathrm{m}$ in length and 0.5-0.8 $\mu \mathrm{m}$ in width (Table 1 and Figure 2) [3]. Cells are Gram-positive and form spherical endospores [3]. Flagella were not observed [3]. Strain $\mathrm{NAL}^{\mathrm{T}}$ was found to grow between $28^{\circ} \mathrm{C}$ and $62^{\circ} \mathrm{C}$ with an optimum at $48^{\circ} \mathrm{C}$ [35]. The upper and lower temperatures for growth were not determined but were predicted to be $10^{\circ} \mathrm{C}$ and $62^{\circ} \mathrm{C}$ [35]. The $\mathrm{pH}$ range for growth was 1.6-2.3 with an optimum at 1.8 [35]. Three strains of $S$. acidophilus have been found to be facultative anaerobes that are able to use $\mathrm{Fe}^{+3}$ as an electron acceptor under anaerobic conditions [4]; but strain $\mathrm{NAL}^{\mathrm{T}}$ was not tested in this study. Strain $\mathrm{NAL}^{\mathrm{T}}$ can grow autotrophically or mixotrophically by oxidizing $\mathrm{Fe}^{+2}$, sulfur, or mineral sulfides or heterotrophically on yeast extract [3]. S. acidophilus and other sulfobacilli have potential applications in biomining. Strain $\mathrm{NAL}^{\mathrm{T}}$ increased the leaching of numerous mineral sulfides [35], however, its sensitivity to low concentrations of metals may limit its usefulness in biomining [35].

\section{Genome sequencing and annotation Genome project history}

This organism was selected for sequencing on the basis of its phylogenetic position [38], and is part of the Genomic Encyclopedia of Bacteria and Archaea project [39]. The genome project is deposited in the Genomes OnLine Database [18] and the complete genome sequence is deposited in GenBank. Sequencing, finishing and annotation were performed by the DOE Joint Genome Institute (JGI). A summary of the project information is shown in Table 2.

\section{Growth conditions and DNA isolation}

S. acidophilus strain NAL ${ }^{\mathrm{T}}$, DSM 10332, was grown in DSMZ medium 709 (Acidomicrobium medium) [40] at $45^{\circ} \mathrm{C}$. DNA was isolated from $0.5-1 \mathrm{~g}$ of cell paste using MasterPure Gram-positive DNA purification kit (Epicentre MGP04100) following the standard protocol as recommended by the manufacturer with modification st/LALM for cell lysis as described in Wu et al. 2009 [39]. DNA is available through the DNA Bank Network [41]. 

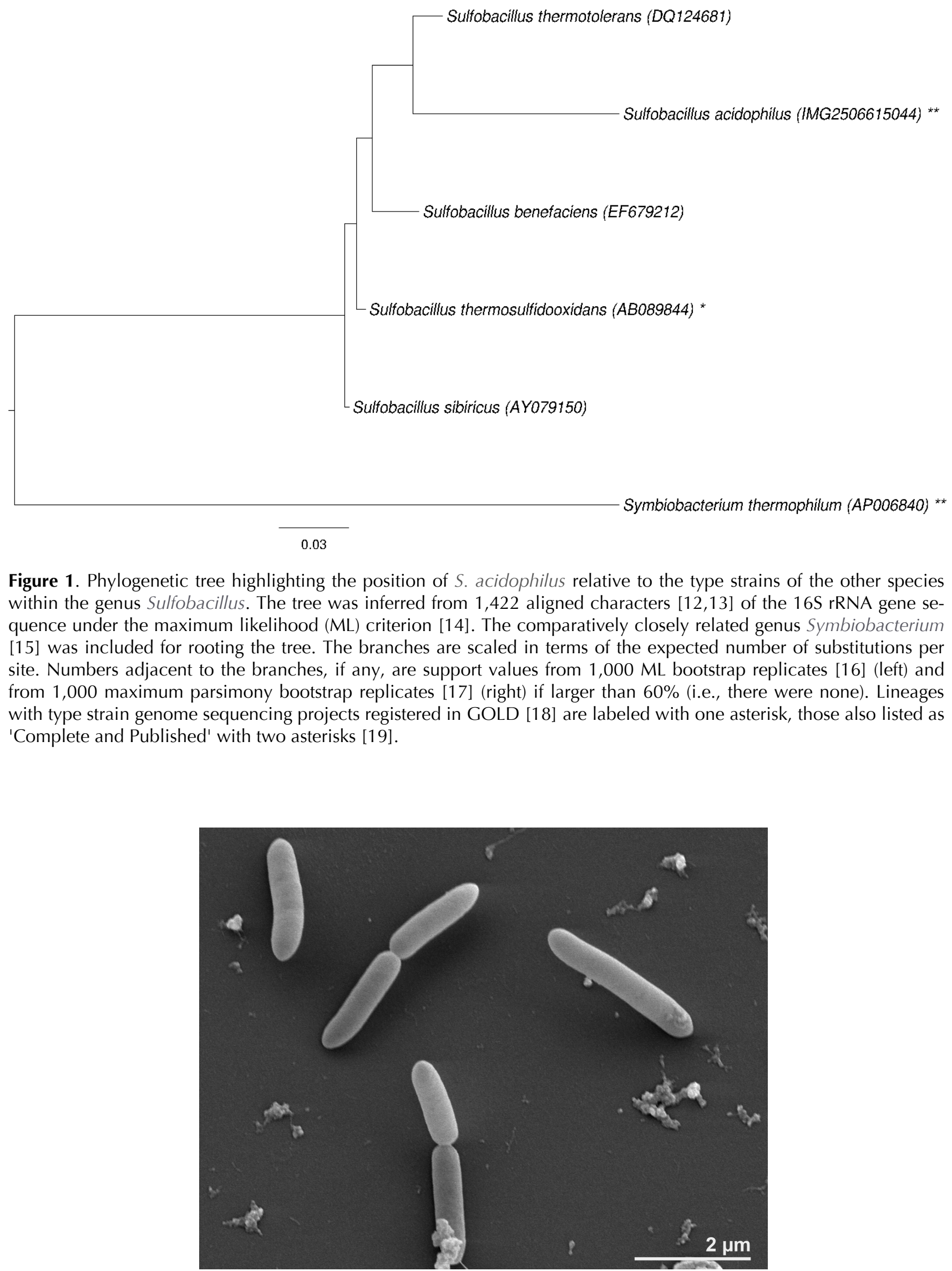

Figure 2. Scanning electron micrograph of $S$. acidophilus $\mathrm{NAL}^{\top}$ 
Table 1. Classification and general features of $S$. acidophilus $\mathrm{NAL}^{\top}$ according to the MIGS recommendations [20] and the NamesforLife database [21].

\begin{tabular}{|c|c|c|c|}
\hline MIGS ID & Property & Term & Evidence code \\
\hline & \multirow{8}{*}{ Current classification } & Domain Bacteria & TAS [22] \\
\hline & & Phylum "Firmicutes" & TAS [23-25] \\
\hline & & Class Clostridia & TAS $[26,27]$ \\
\hline & & Order Clostridiales & TAS $[28,29]$ \\
\hline & & Family "XVII incertae sedis" & TAS $[5,30]$ \\
\hline & & Genus Sulfobacillus & TAS [31-33] \\
\hline & & Species Sulfobacillus acidophilus & TAS $[3,34]$ \\
\hline & & Type strain NAL & TAS [3] \\
\hline & Gram stain & positive & TAS [3] \\
\hline & Cell shape & rods & TAS [3] \\
\hline & Motility & non-motile & NAS \\
\hline & Sporulation & spherical endospores & TAS [3] \\
\hline & Temperature range & not reported & \\
\hline & Optimum temperature & $48^{\circ} \mathrm{C}$ & TAS [35] \\
\hline & Salinity & not reported & \\
\hline \multirow[t]{3}{*}{ MIGS-22 } & Oxygen requirement & facultative anaerobe & TAS [4] \\
\hline & Carbon source & $\mathrm{CO}_{2}$, organic compounds & TAS [3] \\
\hline & Energy metabolism & autotrophic, mixotrophic, heterotrophic & TAS [3] \\
\hline MIGS-6 & Habitat & acidic sulfidic and sulfurous sites & TAS [35] \\
\hline MIGS-15 & Biotic relationship & free-living & TAS [3] \\
\hline \multirow[t]{3}{*}{ MIGS-14 } & Pathogenicity & none & NAS \\
\hline & Biosafety level & 1 & TAS [36] \\
\hline & Isolation & coal spoil heap & TAS [3] \\
\hline MIGS-4 & Geographic location & Alvecote, North Warwickshire, UK & TAS [3] \\
\hline MIGS-5 & Sample collection time & 1988 & TAS [3] \\
\hline MIGS-4.1 & Latitude & 52.638 & TAS [3] \\
\hline MIGS-4.2 & Longitude & -1.641 & TAS [3] \\
\hline MIGS-4.3 & Depth & not reported & \\
\hline MIGS-4.4 & Altitude & not reported & \\
\hline
\end{tabular}

Evidence codes - IDA: Inferred from Direct Assay (first time in publication); TAS: Traceable Author Statement (i.e., a direct report exists in the literature); NAS: Non-traceable Author Statement (i.e., not directly observed for the living, isolated sample, but based on a generally accepted property for the species, or anecdotal evidence). These evidence codes are from the Gene Ontology project [37]. If the evidence code is IDA, then the property was directly observed for a living isolate by one of the authors or an expert mentioned in the acknowledgements. 
Anderson et al.

\begin{tabular}{lll}
\hline \multicolumn{2}{l}{ Table 2. Genome sequencing project information } \\
\hline MIGS ID & Property & Term \\
\hline MIGS-31 & Finishing quality & Finished \\
MIGS-28 & Libraries used & Four genomic libraries: one 454 pyrosequence standard library, \\
& & two 454 PE libraries (6 kb and 10 kb insert size), one Illumina library \\
MIGS-29 & Sequencing platforms & Illumina GAii, 454 GS FLX Titanium \\
MIGS-31.2 & Sequencing coverage & 168.4 $\times$ Illumina; $51.2 \times$ pyrosequence \\
MIGS-30 & Assemblers & Newbler version 2.3-PreRelease-6/30/2009, Velvet 1.0.13, \\
MIGS-32 & Gene calling method & Prodigal 1.4, GenePRIMP \\
& INSDC ID & CP003179 (chromosome) \\
& Genbank Date of Release & CP003180 (plasmid, unnamed) \\
& December 14, 2011 \\
& GOLD ID & Gc02053 \\
& NCBI project ID & 40777 \\
Database: IMG-GEBA & 2506520015 \\
SIGS-13 & Source material identifier & DSM 10332 \\
& Project relevance & Tree of Life, GEBA, biomining \\
\hline
\end{tabular}

\section{Genome sequencing and assembly}

The genome was sequenced using a combination of Illumina and 454 sequencing platforms. All general aspects of library construction and sequencing can be found at the JGI website [42]. Pyrosequencing reads were assembled using the Newbler assembler (Roche). The initial Newbler assembly consisting of 104 contigs in three scaffolds was converted into a phrap [43] assembly by making fake reads from the consensus, to collect the read pairs in the 454 paired end library. Illumina GAii sequencing data (599.7 Mb) were assembled with Velvet [44] and the consensus sequences were shredded into $1.5 \mathrm{~kb}$ overlapped fake reads and assembled together with the 454 data. The 454 draft assembly was based on $143.7 \mathrm{Mb}$ of 454 draft data and all of the 454 paired-end data. Newbler parameters were -consed -a 50 -l 350 -g -m -ml 20. The Phred/Phrap/Consed software package [43] was used for sequence assembly and quality assessment in the subsequent finishing process. After the shotgun stage, reads were assembled with parallel phrap (High Performance Software, LLC). Possible misassemblies were corrected with gapResolution (C. Han, unpublished), Dupfinisher [45], or sequencing cloned bridging PCR fragments with subcloning. Gaps between contigs were closed by editing in Consed, PCR and Bubble PCR primer walks (J.-F. Chang, unpublished). A total of 640 additional reactions and eight shatter libraries were necessary to close gaps and to raise the quality of the finished sequence. Illumina reads were also used to correct potential base errors and increase consensus quality using the software Polisher developed at JGI [46]. The error rate of the completed genome sequence is less than 1 in 100,000 . Together, the combination of the Illumina and 454 sequencing platforms provided $219.6 \times$ coverage of the genome. The final assembly contained 612,059 pyrosequence and 16,626,072 Illumina reads.

\section{Genome annotation}

Genes were identified using Prodigal [47] as part of the Oak Ridge National Laboratory genome annotation pipeline, followed by a round of manual curation using the JGI GenePRIMP pipeline [48]. The predicted CDSs were translated and used to search the National Center for Biotechnology Information (NCBI) nonredundant database, UniProt, TIGR-Fam, Pfam, PRIAM, KEGG, COG, and InterPro databases. Additional gene prediction analysis and functional annotation was performed within the Integrated Microbial Genomes - Expert Review (IMG-ER) platform [49].

\section{Genome properties}

The genome consists of one circular chromosome of $3,472,898 \mathrm{bp}$ and one circular plasmid of $84,933 \mathrm{bp}$ length with an overall $\mathrm{G}+\mathrm{C}$ content of $56.8 \%$ (Table 3 and Figures 3 and 4). Based on coverage of 454 paired ends, the plasmid may be inserted into the chromosome in about half of the population. Of the 3,695 genes predicted, 3,626 are protein-coding genes, and 69 are RNAs; 155 pseudogenes were also identified. The majority of the protein-coding genes $(68.3 \%)$ were assigned a putative function while the remaining ones were annotated as hypothetical proteins. The distribution of genes into COGs functional categories is presented in Table 4. 
Table 3. Genome Statistics

\begin{tabular}{lrr}
\hline Attribute & Value & \% of Total \\
\hline Genome size (bp) & $3,557,831$ & $100.00 \%$ \\
DNA coding region (bp) & $3,106,298$ & $87.31 \%$ \\
DNA G+C content (bp) & $2,019,235$ & $56.75 \%$ \\
Number of replicons & 2 & \\
Extrachromosomal elements & 1 & \\
Total genes & 3,695 & \\
RNA genes & 59 & \\
rRNA operons & 5,626 & $100.00 \%$ \\
Protein-coding genes & 155 & $4.27 \%$ \\
Pseudo genes & 2,475 & $68.26 \%$ \\
Genes with function prediction & 1,896 & $52.29 \%$ \\
Genes in paralog clusters & 2,740 & $75.57 \%$ \\
Genes assigned to COGs & 413 & $11.39 \%$ \\
Genes assigned Pfam domains & 652 & $17.98 \%$ \\
Genes with signal peptides & 910 & $25.10 \%$ \\
Genes with transmembrane helices & & \\
CRISPR repeats & & \\
\hline
\end{tabular}

a) The total is based on either the size of the genome in base pairs or the total number of protein coding genes in the annotated genome.

\section{Insights into the genome sequence Comparative genomics}

While the sequencing of the genome described in this paper was underway, Li et al. from the Third Institute of Oceanography, Xiamen, China published the complete genome sequence of strain TPY [7]. The two genomes differ in size by less than $7,000 \mathrm{bp}$. Here, we take the opportunity to compare the completed genome sequences from these two stains, NAL ${ }^{\mathrm{T}}$ and TPY, both belonging to S. acidophilus. While the biological material for the type stain, $\mathrm{NAL}^{\mathrm{T}}$, is publicly available from the DSMZ open collection for postgenomic analyses, no source of the biological material (MIGS-13 criterion, see Table 2) of strain TPY was provided by Li et al. [7].

To estimate the overall similarity between the genomes of strains NAL ${ }^{\mathrm{T}}$ and TPY (Genbank accession number: CP002901), the GGDC-Genome-toGenome Distance Calculator [50,51] was used. The system calculates the distances by comparing the genomes to obtain HSPs (high-scoring segment pairs) and interfering distances from three formulae (HSP length / total length; identities / HSP length; identities / total length). The comparison of the genomes of strains NAL ${ }^{\mathrm{T}}$ and TPY revealed that $99.65 \%$ of the average of the genome lengths are covered with HSPs. The identity within these HSPs was $99.01 \%$, whereas the identity over the whole genome (counting regions not covered by HSPs as non-identical) was $98.67 \%$. The inferred digital DNA-DNA hybridization values for the two strains are $96.47 \%$ (formula 1 in [51]), 86.08\% (formula 2 in [51]) and $97.05 \%$ (formula 3 in [51]), respectively. These results clearly demonstrate that according to the whole genome sequences of strains NAL ${ }^{\mathrm{T}}$ and TPY, the similarity is very high, supporting the membership of both strains in the same species. 


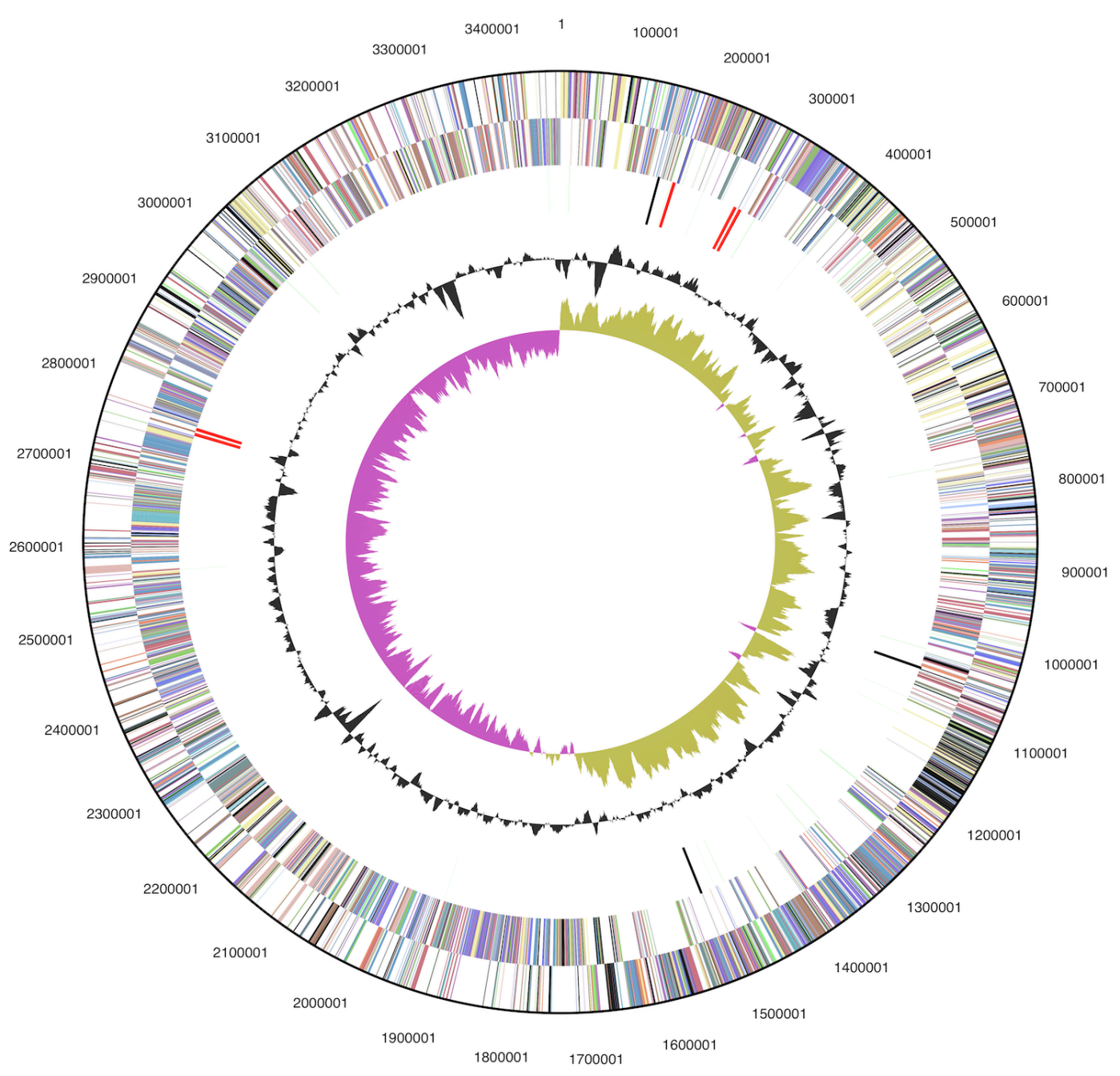

Figure 3. Graphical map of the chromosome. From outside to the center: Genes on forward strand (colored by COG categories), Genes on reverse strand (colored by COG categories), RNA genes (tRNAs green, rRNAs red, other RNAs black), GC content, GC skew.

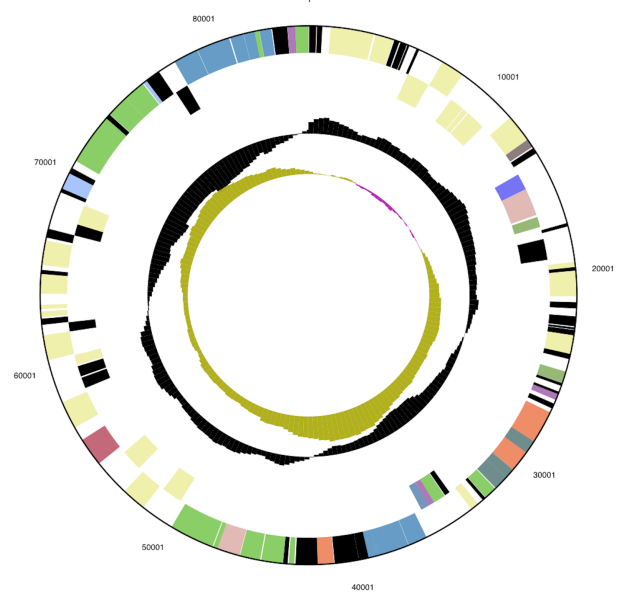

Figure 4. Graphical map of the plasmid. From outside to the center: Genes on forward strand (colored by COG categories), Genes on reverse strand (colored by COG categories), RNA genes (tRNAs green, rRNAs red, other RNAs black), GC content, GC skew. 


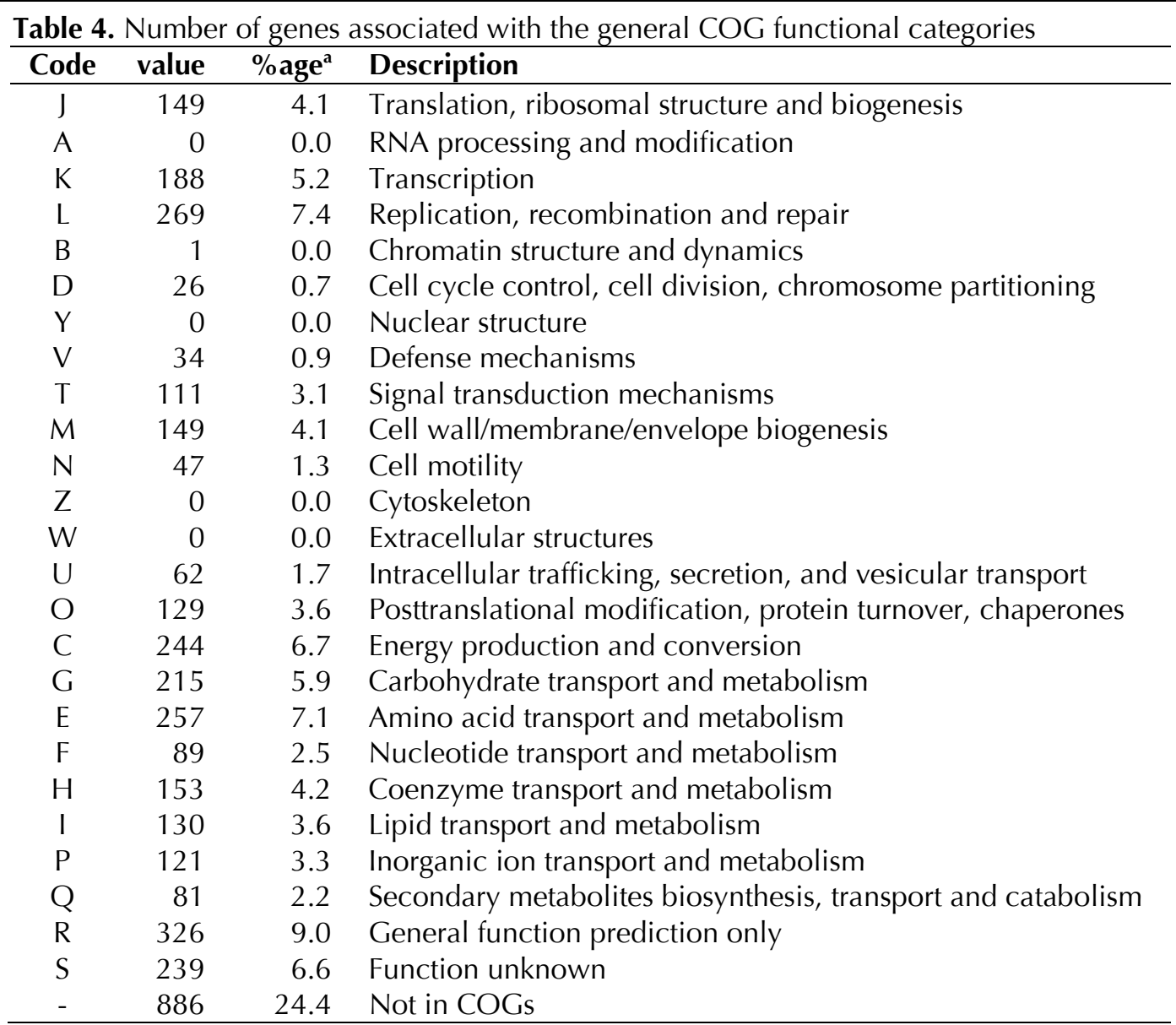

a) The percentage is based on the total number of protein coding genes in the annotated genome.

The comparison of the number of genes belonging to the different COG categories revealed few differences between the genomes of strains $\mathrm{NAL}^{\mathrm{T}}$ and TPY. Strain NAL ${ }^{T}$ has 2,740 genes with COGs assigned, while strain TPY has 2,700. We analyzed the differences in COG assignment between the two strains and found that in almost all cases they could be explained by differences in the gene calls or pseudogene assignment, i.e. in one genome two parts of a pseudogene were called as two separate genes, while in the other genome they were combined into one pseudogene. The only clear case of a difference in gene content between the two strains is the presence of a transposable element consisting of two genes (Sulac_1668, Sulac_1669) disrupting a subunit of a potassium transporter (Sulac_1667) in strain NALT. There were also cases where a gene in one strain was split into two genes in the other strain. For example, Sulac_2178 corresponds to TPY_1983 and TPY1984, and Sulac_0347 corresponds to TPY_0381 and TPY_0382. In both cases the differences are due to a single base indel.
A dot plot showed that there are large blocks of synteny between the two genomes with some rearrangements (data not shown). The genes found on the plasmid in strain $\mathrm{NAL}^{\mathrm{T}}$ are found in two regions of the chromosome in strain TPY. Sulac_3528-3555 corresponds to TPY_0524-0552, while Sulac_35563626 corresponds to TPY_2310-2244. This suggests that in strain TPY, the plasmid was inserted into the chromosome and then split into two pieces.

We analyzed CRISPR repeats with the CRISPR Recognition Tool [52] and found major differences between the two strains. They both have two regions of CRISPR repeats, but the strain TPY repeat regions have 8 and 9 repeats while the strain NAL ${ }^{T}$ repeat regions have 27 and 43 repeats. All of the spacers in the TPY repeat regions are found in $\mathrm{NAL}^{\mathrm{T}}$, but $\mathrm{NAL}^{\mathrm{T}}$ has many additional spacers. This agrees with previous results suggesting that CRISPRs evolve quickly, and differences can be found in closely related strains [53]. 


\section{Acknowledgements}

We would like to gratefully acknowledge the help of Gabriele Gehrich-Schröter for growing S. acidophilus cultures and Susanne Schneider for DNA extraction (both at DSMZ). This work was performed under the auspices of the US Department of Energy Office of Science, Biological and Environmental Research Program, and by the University of California, Lawrence Berkeley National Laboratory under contract No. DE-AC02-

\section{References}

1. Euzéby JP. List of bacterial names with standing in nomenclature: A folder available on the Internet. Int J Syst Bacteriol 1997; 47:590-592. PubMed http://dx.doi.org/10.1099/00207713-47-2-590

2. Johnson DB, Joulian C, d'Hugues P, Hallberg KB. Sulfobacillus benefaciens sp. nov., an acidophilic facultative anaerobic Firmicute isolated from mineral bioleaching operations. Extremophiles 2008; 12:789-798. PubMed http://dx.doi.org/10.1007/s00792-008-0184-4

3. Norris PR, Clark DA, Owen JP, Waterhouse S. Characteristics of Sulfobacillus acidophilus sp. nov. and other moderately thermophilic mineralsulphide-oxidizing bacteria. Microbiology 1996; 142:775-783. PubMed http://dx.doi.org/10.1099/00221287-142-4-775

4. Bridge TAM, Johnson DB. Reduction of soluble iron and reductive dissolution of ferric ironcontaining minerals by moderately thermophilic iron-oxidizing bacteria. Appl Environ Microbiol 1998; 64:2181-2186. PubMed

5. Ludwig W, Schleifer KH, Whitman WB. Revised road map to the phylum Firmicutes. In: Bergey's Manual of Systematic Bacteriology, $2^{\text {nd }}$ ed., vol. 3. De Vos P, Garrity G, Jones D, Krieg NR, Ludwig W, Rainey FA, Schleifer KH, Whitman WB (eds). 2009. Springer-Verlag, New York. pp. 1-13.

6. Golovacheva RS, Karavaiko GI. Sulfobacillus, a new genus of thermophilic sporulating bacteria. Mikrobiologiia 1978; 47:815-822. PubMed

7. Li B, Chen Y, Liu Q, Hu S, Chen X. Complete genome analysis of Sulfobacillus acidophilus strain TPY, isolated from a hydrothermal vent in the Pacific Ocean. I Bacteriol 2011; 193:5555-5556. PubMed http://dx.doi.org/10.1128//B.05684-11

8. Altschul SF, Gish W, Miller W, Myers E, Lipman D. Basic local alignment search tool. J Mol Biol 1990; 215:403-410. PubMed

9. Korf I, Yandell M, Bedell J. BLAST, O'Reilly, Sebastopol, 2003.
05CH11231, Lawrence Livermore National Laboratory under Contract No. DE-AC52-07NA27344, and Los Alamos National Laboratory under contract No. DEAC02-06NA25396, UT-Battelle and Oak Ridge National Laboratory under contract DE-AC05-000R22725, as well as German Research Foundation (DFG) INST 599/1-2.

10. DeSantis TZ, Hugenholtz $P$, Larsen $N$, Rojas $M$, Brodie EL, Keller K, Huber T, Dalevi D, Hu P, Andersen GL. Greengenes, a chimera-checked $16 \mathrm{~S}$ rRNA gene database and workbench compatible with ARB. Appl Environ Microbiol 2006; 72:5069-5072. PubMed http://dx.doi.org/10.1128/AEM.03006-05

11. Porter MF. An algorithm for suffix stripping. Program: electronic library and information systems 1980; 14:130-137.

12. Lee C, Grasso C, Sharlow MF. Multiple sequence alignment using partial order graphs. Bioinformatics 2002; 18:452-464. PubMed http://dx.doi.org/10.1093/bioinformatics/18.3.452

13. Castresana J. Selection of conserved blocks from multiple alignments for their use in phylogenetic analysis. Mol Biol Evol 2000; 17:540-552. PubMed http://dx.doi.org/10.1093/oxfordjournals.molbev.a $\underline{026334}$

14. Stamatakis A, Hoover P, Rougemont J. A rapid bootstrap algorithm for the RAxML web-servers. Syst Biol 2008; 57:758-771. PubMed http://dx.doi.org/10.1080/10635150802429642

15. Yarza P, Ludwig W, Euzéby J, Amann R, Schleifer $\mathrm{KH}$, Glöckner FO, Rosselló-Móra R. Update of the All-Species Living Tree Project based on 16S and 23S rRNA sequence analyses. Syst Appl Microbiol 2010; 33:291-299. PubMed http://dx.doi.org/10.1016/j.syapm.2010.08.001

16. Pattengale ND, Alipour M, Bininda-Emonds ORP, Moret BME, Stamatakis A. How many bootstrap replicates are necessary? Lect Notes Comput Sci 2009; 5541:184-200. http://dx.doi.org/10.1007/978-3-642-02008-7_13

17. Swofford DL. PAUP*: Phylogenetic Analysis Using Parsimony (*and Other Methods), Version 4.0 b10. Sinauer Associates, Sunderland, 2002.

18. Pagani I, Liolios K, Jansson J, Chen IM, Smirnova T, Nosrat B, Markowitz VM, Kyrpides NC. The Genomes OnLine Database (GOLD) v.4: status of 
genomic and metagenomic projects and their associated metadata. Nucleic Acids Res 2012;

40:D571-D579. PubMed http://dx.doi.org/10.1093/nar/gkr1100

19. Ueda K, Yamashita A, Ishikawa J, Shimada M, Watsuji TO, Morimura K, Ikeda H, Hattori M, Beppu T. Genome sequence of Symbiobacterium thermophilum, an uncultivatable bacterium that depends on microbial commensalism. Nucleic Acids Res 2004; 32:4937-4944. PubMed http://dx.doi.org/10.1093/nar/gkh830

20. Field D, Garrity G, Gray T, Morrison N, Selengut J, Sterk P, Tatusova T, Thomson N, Allen MJ, Angiuoli SV, et al. The minimum information about a genome sequence (MIGS) specification. Nat Biotechnol 2008; 26:541-547. PubMed http://dx.doi.org/10.1038/nbt1360

21. Garrity G. NamesforLife. BrowserTool takes expertise out of the database and puts it right in the browser. Microbiol Today 2010; 37:9.

22. Woese CR, Kandler O, Wheelis ML. Towards a natural system of organisms: proposal for the domains Archaea, Bacteria, and Eucarya. Proc Natl Acad Sci USA 1990; 87:4576-4579. PubMed http://dx.doi.org/10.1073/pnas.87.12.4576

23. Gibbons NE, Murray RGE. Proposals Concerning the Higher Taxa of Bacteria. Int I Syst Bacteriol 1978; 28:1-6. http://dx.doi.org/10.1099/00207713-28-1-1

24. Garrity GM, Holt JG. The Road Map to the Manual. In: Garrity GM, Boone DR, Castenholz RW (eds), Bergey's Manual of Systematic Bacteriology, Second Edition, Volume 1, Springer, New York, 2001, p. 119-169.

25. Murray RGE. The Higher Taxa, or, a Place for Everything...? In: Holt JG (ed), Bergey's Manual of Systematic Bacteriology, First Edition, Volume 1, The Williams and Wilkins Co., Baltimore, 1984, p. 31-34.

26. List Editor. List of new names and new combinations previously effectively, but not validly, published. List no. 132. Int / Syst Evol Microbiol 2010; 60:469-472. http://dx.doi.org/10.1099/ijs.0.022855-0

27. Rainey FA. Class II. Clostridia class nov. In: De Vos P, Garrity G, Jones D, Krieg NR, Ludwig W, Rainey FA, Schleifer KH, Whitman WB (eds), Bergey's Manual of Systematic Bacteriology, Second Edition, Volume 3, Springer-Verlag, New York, 2009, p. 736.
28. Skerman VBD, McGowan V, Sneath PHA. Approved Lists of Bacterial Names. Int J Syst Bacteriol 1980; 30:225-420. http://dx.doi.org/10.1099/00207713-30-1-225

29. Prévot AR. In: Hauderoy P, Ehringer G, Guillot G, Magrou. J., Prévot AR, Rosset D, Urbain A (eds), Dictionnaire des Bactéries Pathogènes, Second Edition, Masson et Cie, Paris, 1953, p. 1-692.

30. da Costa MS, Rainey FA. Family II. Alicyclobacillaceae fam. nov. In: De Vos P, Garrity G, Jones D, Krieg NR, Ludwig W, Rainey FA, Schleifer KH, Whitman WB (eds), Bergey's Manual of Systematic Bacteriology, Second Edition, Volume 3, Springer-Verlag, New York, 2009, p. 229.

31. List Editor. Validation List no. 36. Validation of the publication of new names and new combinations previously effectively published outside the IJSB. Int J Syst Bacteriol 1991; 41:178-179. http://dx.doi.org/10.1099/00207713-41-1-178

32. Golovacheva RS, Karavaiko GI. Sulfobacillus, a new genus of thermophilic sporulating bacteria. Mikrobiologiia 1978; 47:815-822. PubMed

33. Johnson DB, Joulian C, d'Hugues P, Hallberg KB. Sulfobacillus benefaciens sp. nov., an acidophilic facultative anaerobic Firmicute isolated from mineral bioleaching operations. Extremophiles 2008; 12:789-798. PubMed http://dx.doi.org/10.1007/s00792-008-0184-4

34. List Editor. Validation List no. 59. Validation of the publication of new names and new combinations previously effectively published outside the IJSB. Int J Syst Bacteriol 1996; 46:1189-1190. http://dx.doi.org/10.1099/00207713-46-4-1189

35. Watling HR, Perrot FA, Shiers DW. Comparison of selected characteristics of Sulfobacillus species and review of their occurrence in acidic and bioleaching environments. Hydrometallurgy 2008; 93:57-65. http://dx.doi.org/10.1016/j.hydromet.2008.03.001

36. BAuA. Classification of Bacteria and Archaea in risk groups. TRBA 466. p. 227. Bundesanstalt für Arbeitsschutz und Arbeitsmedizin, Germany. 2010.

37. Ashburner M, Ball CA, Blake JA, Botstein D, Butler H, Cherry JM, Davis AP, Dolinski K, Dwight SS, Eppig JT, et al. Gene Ontology: tool for the unification of biology. Nat Genet 2000; 25:25-29. PubMed http://dx.doi.org/10.1038/75556

38. Klenk HP, Göker M. En route to a genome-based classification of Archaea and Bacteria? Syst Appl 
Anderson et al.

Microbiol 2010; 33:175-182. PubMed

http://dx.doi.org/10.1016/j.syapm.2010.03.003

39. Wu D, Hugenholtz $P$, Mavromatis $K$, Pukall R, Dalin E, Ivanova NN, Kunin V, Goodwin L, Wu $\mathrm{M}$, Tindall BJ, et al. A phylogeny-driven genomic encyclopaedia of Bacteria and Archaea. Nature 2009; 462:1056-1060. PubMed

http://dx.doi.org/10.1038/nature08656

40. List of growth media used at DSMZ: http//www.dsmz.de/catalogues/cataloguemicroorganisms/culture-technology/list-of-mediafor-microorganisms.html.

41. Gemeinholzer B, Dröge $G$, Zetzsche $H$, Haszprunar G, Klenk HP, Güntsch A, Berendsohn WG, Wägele JW. The DNA Bank Network: the start from a German initiative. Biopreserv Biobank 2011; 9:51-55.

http://dx.doi.org/10.1089/bio.2010.0029

42. JGI website. http://www.jgi.doe.gov.

43. The Phred/Phrap/Consed software package. http://www.phrap.com.

44. Zerbino DR, Birney E. Velvet: algorithms for de novo short read assembly using de Bruijn graphs. Genome Res 2008; 18:821-829. PubMed http://dx.doi.org/10.1101/gr.074492.107

45. Han C, Chain P. Finishing repeat regions automatically with Dupfinisher. In: Proceeding of the 2006 international conference on bioinformatics \& computational biology. Arabnia HR, Valafar $\mathrm{H}$ (eds), CSREA Press. June 26-29, 2006: 141-146.

46. Lapidus A, LaButti K, Foster B, Lowry S, Trong S, Goltsman E. POLISHER: An effective tool for using ultra short reads in microbial genome assembly and finishing. AGBT, Marco Island, FL, 2008.
47. Hyatt D, Chen GL, LoCascio PF, Land ML, Larimer FW, Hauser LJ. Prodigal: prokaryotic gene recognition and translation initiation site identification. BMC Bioinformatics 2010; 11:119. PubMed http://dx.doi.org/10.1186/1471-2105-11-119

48. Pati A, Ivanova NN, Mikhailova N, Ovchinnikova G, Hooper SD, Lykidis A, Kyrpides NC.

GenePRIMP: a gene prediction improvement pipeline for prokaryotic genomes. Nat Methods 2010; 7:455-457. PubMed http://dx.doi.org/10.1038/nmeth.1457

49. Markowitz VM, Ivanova NN, Chen IMA, Chu K, Kyrpides NC. IMG ER: a system for microbial genome annotation expert review and curation. Bioinformatics 2009; 25:2271-2278. PubMed http://dx.doi.org/10.1093/bioinformatics/btp393

50. Auch AF, Von Jan M, Klenk HP, Göker M. Digital DNA-DNA hybridization for microbial species delineation by means of genome-to-genome sequence comparison. Stand Genomic Sci 2010; 2:117-134. PubMed http://dx.doi.org/10.4056/sigs.531120

51. Auch AF, Klenk HP, Göker M. Standard operating procedure for calculating genome-to-genome distances based on high-scoring segment pairs. Stand Genomic Sci 2010; 2:142-148. PubMed http://dx.doi.org/10.4056/sigs.541628

52. Bland C, Ramsey TL, Sabree F, Lowe M, Brown K, Kyrpides NC, Hugenholtz P. CRISPR recognition tool (CRT): a tool for automatic detection of clustered regularly interspaced palindromic repeats. BMC Bioinformatics 2007; 8:209. PubMed http://dx.doi.org/10.1186/1471-2105-8-209

53. Tyson GW, Banfield JF. Rapidly evolving CRISPRs implicated in acquired resistance of microorganisms to viruses. Environ Microbiol 2008; 10:200207. PubMed 